\title{
Hepatic Metastatic GIST: Diagnostic and Therapeutic Difficulties in Souro Sanou Teaching Hospital in Bobo Dioulasso, Burkina Faso
}

\author{
Ollo Roland Somé ${ }^{*}$, Ida Aïda Tankoano¹, Mali Koura1, Valentin Konségré1, Damien Konkobo², \\ Jérôme Koulidiaty ${ }^{3}$ \\ ${ }^{1}$ Institut Supérieur des Sciences de la Santé of Nazi Boni University, Bobo-Dioulasso, Burkina Faso \\ ${ }^{2}$ Université Cheick Anta Diop, Hospital Aristide Le Dantec, Dakar, Sénégal \\ ${ }^{3}$ Université Ouaga 1 Joseph Ki Zerbo, Ouagadougou, Burkina Faso \\ Email: ^som_roll@yahoo.fr
}

How to cite this paper: Somé, O.R., Tankoano, I.A., Koura, M., Konségré, V., Konkobo, D. and Koulidiaty, J. (2018) Hepatic Metastatic GIST: Diagnostic and Therapeutic Difficulties in Souro Sanou Teaching Hospital in Bobo Dioulasso, Burkina Faso. Open Journal of Gastroenterology, 8, 455-462.

https://doi.org/10.4236/ojgas.2018.812047

Received: November 25, 2018

Accepted: December 24, 2018

Published: December 27, 2018

Copyright ( $\odot 2018$ by authors and Scientific Research Publishing Inc. This work is licensed under the Creative Commons Attribution International License (CC BY 4.0).

http://creativecommons.org/licenses/by/4.0/ c) (i) Open Access

\begin{abstract}
Introduction: Gastrointestinal stromal tumor (GIST) is the most common non-epithelial, mesenchymal tumor of the digestive tract. Targeted therapy has improved the prognosis but the diagnosis must be accurate before using the existing drugs. Aim: To report the diagnostic difficulty in our context through the clinical polymorphism and define the position of targeted therapy in the management of GIST metastatic stomach. Casuistry: We report 3 cases of gastric GIST with liver metastasis in different circumstances of discovery. Patients were 21,45 , and 73 years old. Discovery circumstances were respectively digestive hemorrhage, severe clinical anemia, abdominal tumor and gastric tumor. There was hepatic metastasis in the three cases. The three patients received Imatinib treatment, adjuvant treatment for the first two cases, and neo-adjuvant treatment for the third case, with a very good clinical response and CT response on metastases. Conclusion: Because of their rarity, GISTs are often difficult to diagnose, and necessarily require immunohistochemistry which is not available in our work context. The effectiveness of targeted therapy even on metastasis needs a rigorous diagnostic approach to improve patient survival.
\end{abstract}

\section{Keywords}

Gastric Stromal Tumors, Metastases, Imatinib, Surgery, Bobo Dioulasso

\section{Introduction}

Gastrointestinal stromal tumor (GIST) is the most frequent mesenchymal tumor 
of the digestive tract with $0.1 \%$ to $3 \%$ of all gastrointestinal cancers [1] [2] [3] [4].

Gastric location is the most common site, followed by the small intestine, colon, rectum, and esophagus [2] [3] [5]. Gastric GISTs have variable clinical expression [2] [3] [4]. From non-painful form with low metastatic risk, some may remain stable for years whereas others, very symptomatic, progress rapidly and already metastasized at the time of the diagnosis [2] [6]. Local forms treatment is essentially based on surgery R0 [2] [5], however advanced gist (metastatic and/or unresectable from the outset) has an improved prognosis by the addition of the Imatinib, and treatment target of the $\mathrm{CD} 117$ receptor expressed by these tumors [1] [2] [4].

We report three cases of gastric GIST metastasized to the liver to show the importance of the rigorous diagnostic procedure and the contribution of the targeted treatment to tumors whose prognosis is often considered pejorative from the beginning in our context.

\section{Case 1}

A21-year-oldfemale student, consulted in cancer unit in October 2015, referred by the gastroenterology department for Gastric Tumor. She had history of gastric ulcer diagnosed three months before consultation by upper GIT endoscopy and was receiving a treatment. No malignant cells or helicobacter Pylori were found on histology. Because of persistency of symptoms, a second endoscopy was requested and described a bombing tumor with a central crater in the lumen of the small gastric curvature (Figure 1(a)). A histology of a biopsy taken from this indurated lesion was not contributing. Further investigations revealed hepatic localization of secondary metastasis on segment II and IV. Due to the severity of anemia at $5 \mathrm{~g} / \mathrm{dl}, 4 / 5$ gastrectomy was performed on 19/10/2015 and took out a tumor of the small curvature with parietal development in the gastric lumen without breaking the mucosa (Figure 1(b)). The Post-operative evolution was non-complicated and the patient was discharged after 6 days. Weight gain and disappearance of anemia were noted after the surgery. The histology of the operative specimen was compatible with a gastrointestinal stromal tumor (Figure 1(c), Figure 1(d)). The immunohistochemistry investigation performed confirms the diagnosis with CD34 labeling and CD117. An abdominopelvic CT scan of 29/01/2016 before the start of the treatment found a new hepatic location of segment IV (Figure 1(e)). An adjuvant palliative treatment by Imatinib was instituted since February 2016. After 21 months of treatment, a good clinical evolution was noted and a CT scan showed a near complete resolution of hepaticlesions (Figure 1(f)).

\section{Case 2}

A 72 years old, housewife, was received in cancer consulting unit in September 2015 for huge mass occupying the entire abdomen with dyspepsia and weight 


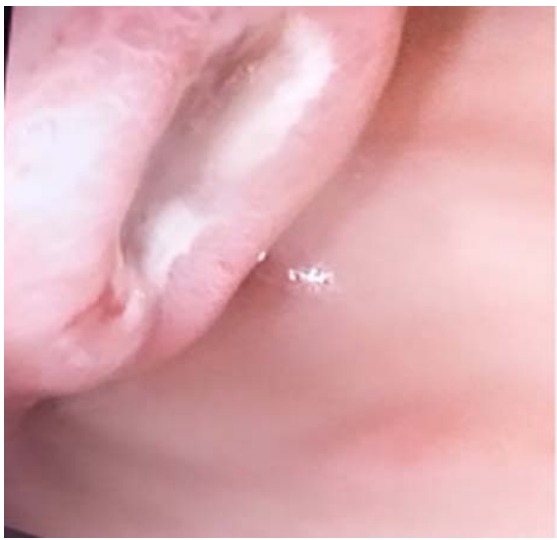

(a)

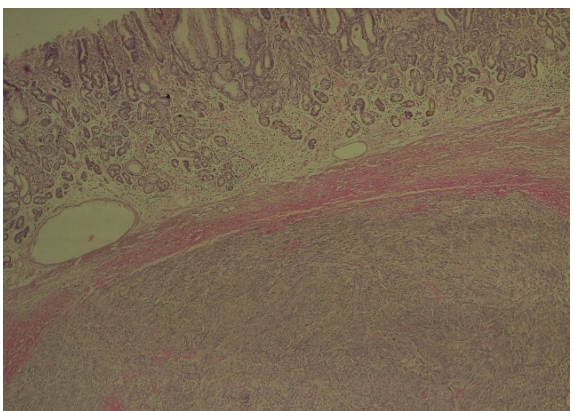

(c)

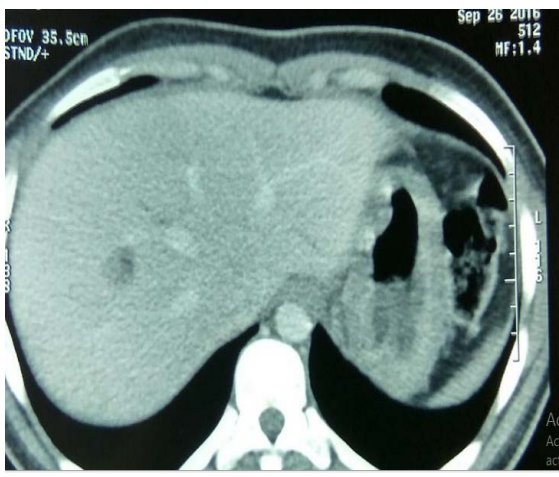

(e)

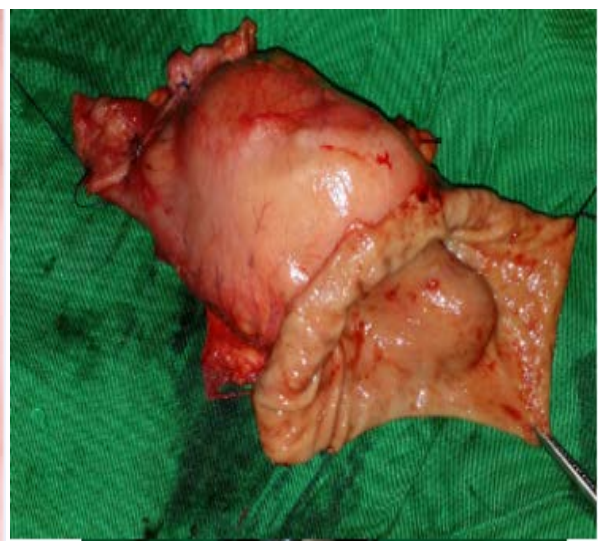

(b)

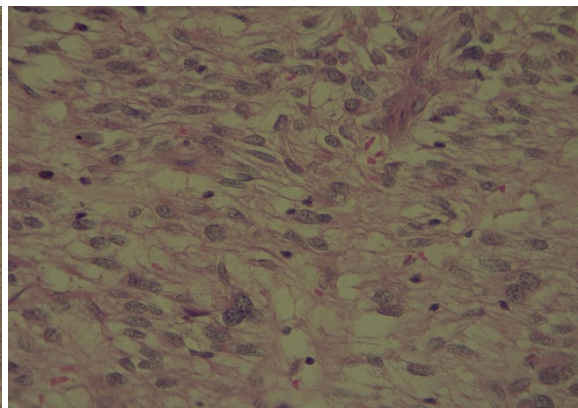

(d)

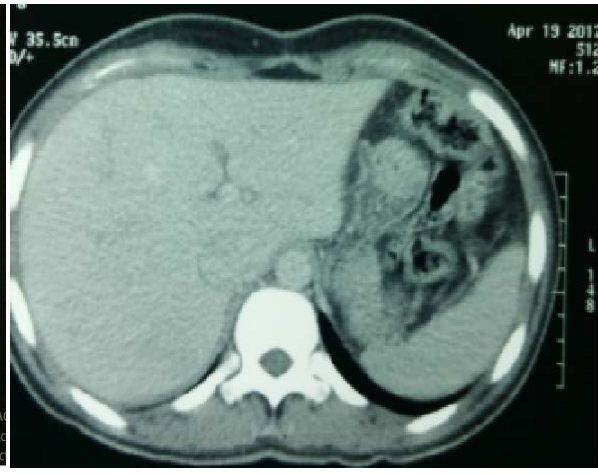

(f)

Figure 1. (a) Endoscopic image of the tumor lesion non-ulcerated crater form that bomb in the light of the small gastric curvature; (b) Part of gastrectomy 4/5 is performed with a tumor depends on the small curvature with bulging wall development in the gastric lumen without breaking the mucosa; (c) Histological samples stained with Hemein-eosin. Low magnification: Gastric wall with sub-mucosal tumor proliferation, well limited and encapsulated; (d) Histological samples stained with Hemein-eosin. Magnification 400: cellular details, large nuclei and sometimes nucleoli; (e) Abdominopelvic CT showing the evolution of the hepatic lesion of the segment IV before treatment; and (f) After 7 months of treatment with Imatinib.

loss for more than a year. There was a previous history of epigastric pain. The abdominal examination found the mass mainly located in the left quadrant with no evidence of ascite. The abdominal CT scan described a tissue-like mass predominantly marked by areas of necrosis (hypodensity) measuring $15 \mathrm{~cm} \times 10$ 
$\mathrm{cm}$, sheathing the spleen without invading it (Figure 2(a)), and associated with multiple mesenteric, pre-pancreatic adenopathies and hepatic lesions secondary to the segment VI. This tumor evoked first a retroperitoneal lymphoma or sarcoma

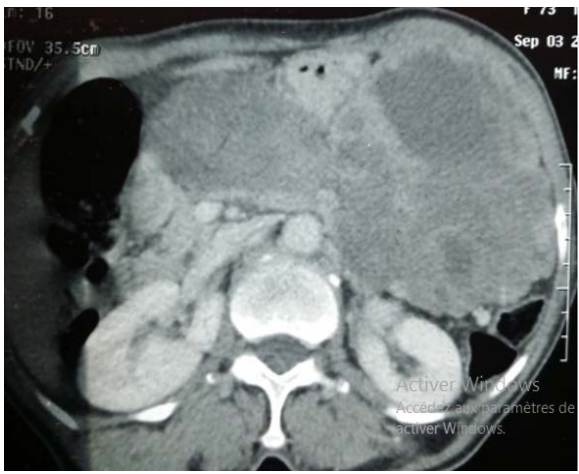

(a)

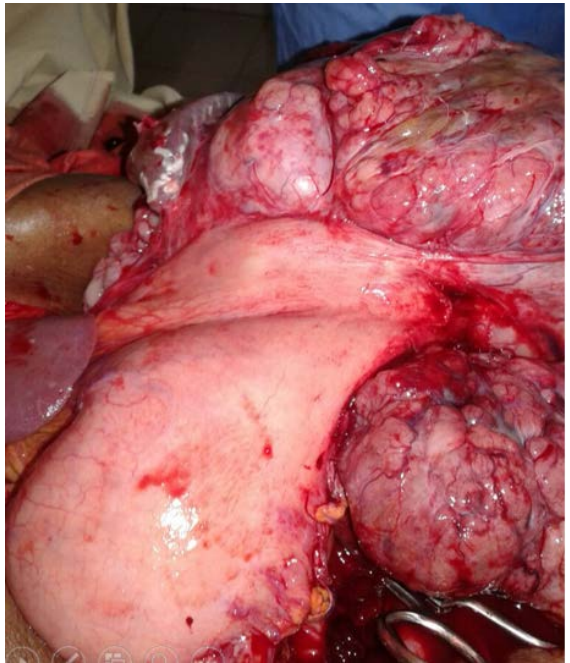

(c)

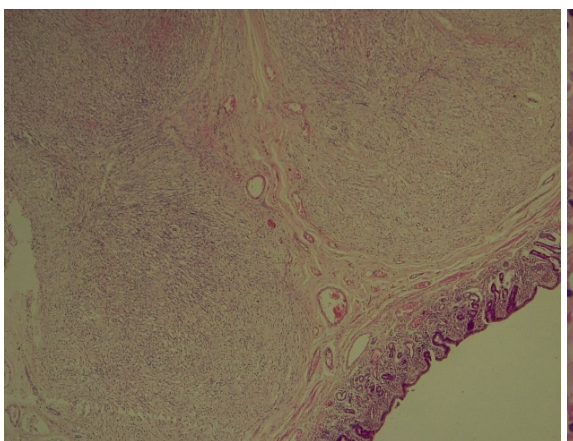

(e)

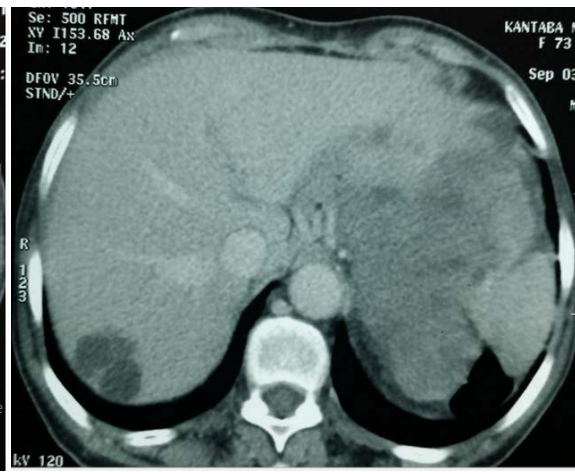

(b)

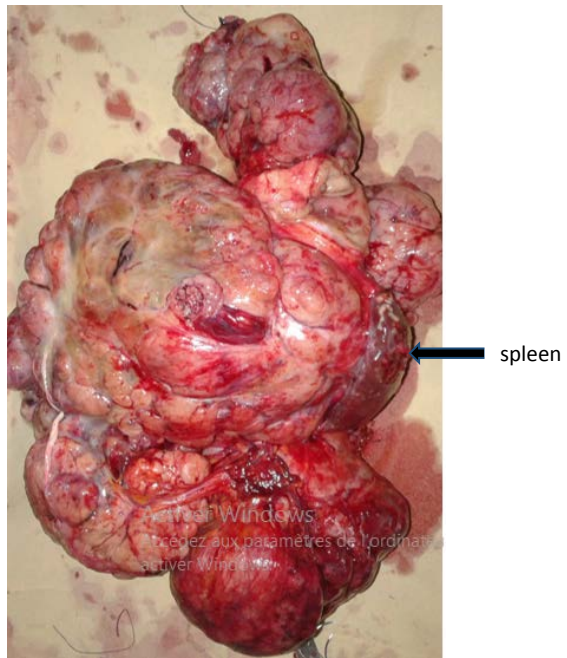

(d)

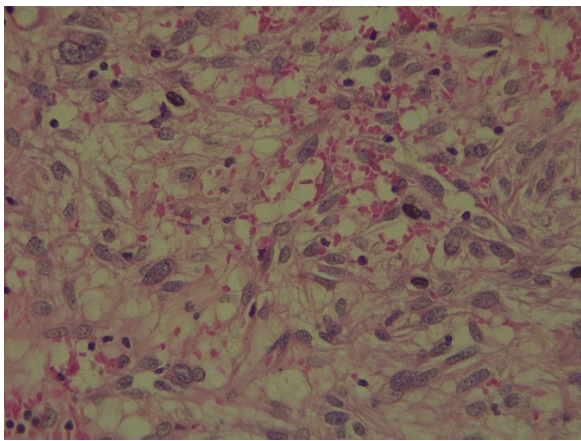

(f)

Figure 2. (a) Pre-pancreatic mass engulfing the spleen without invading it $25 \times 30 \mathrm{~cm}$ evoking Lymphoma (a) with intrahepatic metastasis (b); (c) In surgical exploration there is an irregular fleshy bulky mass encapsulated in several places and invading the large gastric curvature and the splenic hila; (d) That explains the partial atypic spleno-gastrectomy (operative specimen); (e) Low magnification: nodular proliferation of mucosa fasciculate architecture; (f) Magnification 400 showing cellular details: large fusiform, hyperchromatic nuclei and nucleoli. 
with metastasis to the liver (Figure 2(b)). Ultrasound guided biopsy of this accessible mass was made and suspected a benign fibrous lesion. A laparotomy performed in September 2016 (i.e. 1 year after initial consultation) found a voluminous irregular and fleshy mass, encapsulated in some areas, invading the large gastric curvature (Figure 2(c)) and the splenic hila, explaining atypical partial spleno-gastrectomy (Figure 2(d)). The anatomo-pathology examination identified aGIST (Figure 2(e), Figure 2(f)) with positivity of CD117 receptors. Treatment with Imatinib $400 \mathrm{mg}$ started in November 2016.

Digestive intolerance at the beginning was noted and a control after a year of treatment noted a clear clinical improvement with weight recovery and regression of metastatic lesions.

\section{Case 3}

A 44-year-old man, consulted in October 2015 for abdominal pain, severe anaemia treated by multiple transfusion without success. He had a previous history of iterative laparotomy in 2014 for attempted extirpation of an abdominalmass. He had multiple blood transfusions. An endoscopy was performed and showed a voluminous burgeoning tumour mass occupying the antrum and the greater curvature of the stomach. The histology of the biopsy was in favour of proliferation fusiform cells with inflammatory necrotic tissues (Figure 3(a), Figure 3(b)). Immunohistochemical complement confirmed gastrointestinal stromal tumour expressing CD117 but not expressing actin or PS 100. Further investigations found multiple hepatic lesions. The general state gradually deteriorated but remains 1 - 2 of WHO classification. Neo adjuvant treatment with Imatinib started in January 2016. After 12 months of treatment there was a complete clinical resolution of the mass, anaemia improved to $10 \mathrm{~g} / \mathrm{dl}$ and weight gain was also noted.

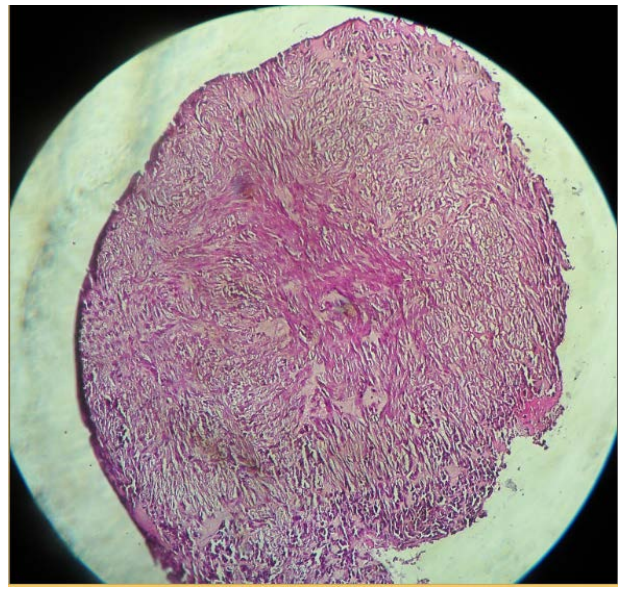

(a)

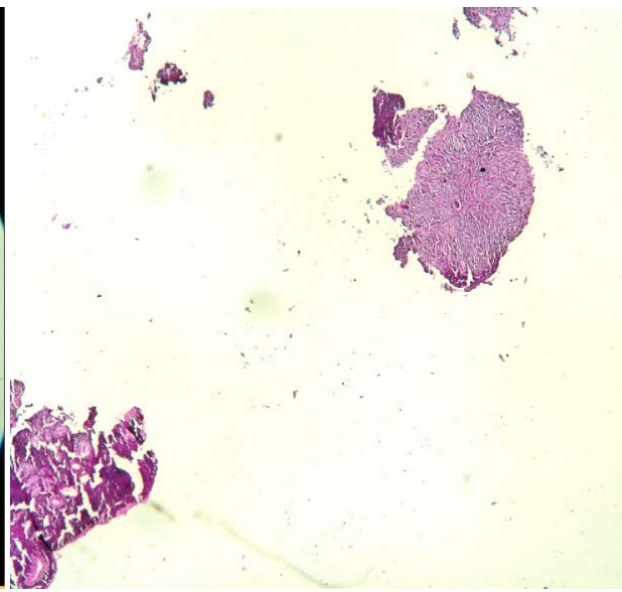

(b)

Figure 3. (a)-(b) Spindle cell proliferation accompanied by necrotic reworking inflammatory. Immunohistochemical complement confirms stromal tumor Gastro-intestinal expressing CD117 but do not express actin or PS 100. 


\section{Discussion}

GISTs are rare pathologies since they represent $0.1 \%$ to $3 \%$ digestive cancers and $10 \%$ sarcomas [1] [2] [3] [4]. A systematic review [2] from 2000 to 2014 reported 13,550 cases from 19 countries and gastric localization accounted for $55.6 \%$ of cases. In our series, they occur at variable ages as reported [2] with extremes of 10 to 100 years. No sex specificity was noted.

We reported three cases of hepatic metastasis from GIST in the same year. That demonstrates that the pathology is relatively rare in our working context. Actually, the frequency may be underestimated due to the diagnostic difficulties [7]. One of the causes of this difficulty is the delay in consultation, related to the variability of symptoms. Dyspepsia is found in almost all cases at the beginning. In advanced forms, anaemia or bulky mass are the most common manifestations found in other series [1] [2] [3] [4]. Nevertheless, the incidental diagnosis was reported in $18.7 \%$ of cases [2]. In our context the delay diagnosis can be explained by unavailability of endoscopy (poorly equipped centres), available only in the two major cities of the country and also the lack of compliance of patients who have the fear of the procedure.

Sometimes, the lesion has an exotic luminal development and can spare the gastric mucosa, and then biopsies may not be contributory. However, the typical endoscopic picture can strongly suspect the diagnosis and requires the indication of atypical partial gastrostomy for diagnostic and therapeutic purpose in some cases, after multidisciplinary consultations [2]. Indeed in one of our cases, endoscopic biopsy performed twice did not allow the diagnosis. The typical image was not enough for the diagnosis in the absence of a concerted opinion. The surgery was needed before repeated severe anaemia despite multiple blood transfusions.

The diagnosis delay can be also connected with the misdiagnosis in front of the big size of the mass suggestive of retro-peritoneal sarcoma or lymphoma. Echo-guided biopsy that we carried out for this case did not allow to make the diagnosis because it found a benign fibrous lesion; endoscopy was not requested due to lack of gastric symptoms. The absence of immunohistochemical study contributes to the underestimated frequency. The diagnosis was confirmed in our three cases because immunohistochemistry was performed in France by Cerba laboratory in Paris and the bill was supported by patients (100,000 CFA francs or 150 Euros). The phenotypic expression was the presence of receptors CD117 or Kit found in $75 \%$ to $85 \%$ of cases in the literature in cases against PDGFRA receptors found in $5 \%$ to $10 \%$ of cases [6] [8]. The evolution to metastases is frequent since $15 \%$ to $50 \%$ of the cases are metastatic at the time of diagnosis. In one of our cases, general state OMS stage 1 - 2 contrasted with the chronic evolution of the huge mass and hepatic metastasis ( $\mathrm{Ki}, \mathrm{MMu}$ low). This further proves the insidious nature, the variability of clinical presentation of this pathology. Hepatic metastases are classically described [2] [9].

In the absence of metastasis, R0 curative resection of lesions larger than $2 \mathrm{~cm}$ 
is the rule. Advances in molecular research have allowed the identification of mutation activating tyrosine kinase-related Kit or PDGFRA receptors in the mutagenesis of Cajal cells [4]. Therefore the addition of the molecule (Imatinib, monoclonal antibody) inhibiting this pathway signal, has radically changed the prognosis, even for metastatic GISTs [10]. Indeed overall survival goes from 41\% without Imatinib to $72 \%$ with Imatinib or $85 \%$ for the Imatinib arm and resection R0 [2]. The effectiveness of the contribution of Imatinib in neoadjuvant treatment in the management of GISTs locally advanced unresectable from the outset is demonstrated in several series [4] [5]. The cost of treatment remains expensive for our poor patients in Burkina Faso without health insurance. Nevertheless, because of North-South cooperation, our patients have been included in the GIPAP program and received this treatment. We found a good clinical and CT scan response and in all three cases. However resection of residual tumor in one case, the patient refused the third surgery because he felt cured. As for the tumour response on Hepatic metastasis, we find like other authors a clinical response almost complete on these locations [4] [9] [11]. The necessity of metastasectomy is still being discussed, without a consensus [2] [12]. While waiting for conclusive randomized studies, some studies seem to point prolonged survival associated with resection R0 coupled with intake of Imatinib [4]. Because of high risk of secondary resistance to Imatinib due to second mutations described by some authors [10] [11] resection of a persistent metastasis would be indicated as well as the tumour residue of more than $2 \mathrm{~cm}$ after neoadjuvant treatment.

\section{Conclusion}

Gastric Gist is the most common digestive mesenchymal tumour. It has to be kept in mind before any gastrointestinal bleeding and gastric mass or bulky meso-colic with non-contributory endoscopic biopsy. The diagnosis of certainty by Immunohistochemistry is necessary in view of the interest of Imatinib in taking metastatic forms or unresectable from the outset.

\section{Patients Consent}

The patients have given their consent for the Case reports to be published.

\section{Acknowledgements}

We would like to thank the Glivec Foundation for free patient care regarding access to Imatinib.

\section{Conflicts of Interest}

The authors declare no conflict of interest.

\section{References}

[1] Keung, E.Z. and Raut, C.P. (2017) Management of Gastrointestinal Stromal Tu- 
mors. Surgical Clinics of North America, 97, 437-452.

https://doi.org/10.1016/j.suc.2016.12.001

[2] Søreide, K., Sandvik, O.M., Søreide, J.A., Giljaca, V., Jureckova, A. and Bulusu, V.R. (2016) Global Epidemiology of Gastrointestinal Stromal Tumors (GIST): A Systematic Review of Population-Based Cohort Studies. Cancer Epidemiology, 40, 39-46. https://doi.org/10.1016/j.canep.2015.10.031

[3] Lai, E.C.H., Lau, S.H.Y. and Lau, W.Y. (2012) Current Management of Gastrointestinal Stromal Tumors-A Comprehensive Review. International Journal of Surgery, 10, 334-340. https://doi.org/10.1016/j.ijsu.2012.05.007

[4] Valsangkar, N., Sehdev, A., Misra, S., Zimmers, T.A., O’Neil, B.H. and Koniaris, L.G. (2015) Current Management of Gastrointestinal Stromal Tumors: Surgery, Current Biomarkers, Mutations, and Therapy. Surgery, 158, 1149-1164. https://doi.org/10.1016/j.surg.2015.06.027

[5] Ford, S.J. and Gronchi, A. (2016) Indications for Surgery in Advanced/Metastatic GIST. European Journal of Cancer, 63, 154-167.

https://doi.org/10.1016/j.ejca.2016.05.019

[6] Rubin, B.P., Heinrich, T.M. and Corless, C.L. (2007) Gastrointestinal Stromal Tumor. The Lancet, 369, 1731-1741. https://doi.org/10.1016/S0140-6736(07)60780-6

[7] Somda, K.S., Ouedraogo, A.S., Coulibaly, A., Koulidiati, J., Bambara, A.H., Nikiema, R., Sombié, A.R., Serme, A.K. and Bougouma, A. (2016) Tumeur Gastrique Stromale. A propose de 02 cas. Médecine d'Afrique Noire, 63.

[8] Zeichner, S.B., Goldstein, D.A., Kohn, C. and Flowers, C.R. (2017) Cost-Effectiveness of Precision in Gastrointestinal Stromal Tumor and Gastric Adenocarcinoma. Journal of Gastrointestinal Oncology, 8, 513-523. https://doi.org/10.21037/jgo.2016.04.03

[9] Rajendra, R., Pollack, S.M. and Jones, R.L. (2013) Management of Gastrointestinal Stromal Tumors. Future Oncology, 9, 193-206.

https://doi.org/10.2217/fon.12.178

[10] Nilsson, B., Nilsson, O. and Ahlman, H. (2009) Treatment of Gastrointestinal Stromal Tumors: Imatinib, Sunitinib-And Then? Expert Opinion on Investigational Drugs, 18, 457-468. https://doi.org/10.1517/13543780902806400

[11] Cesne, A., Ray-Coquard, I., Bui, B.N., Adenis, A., Rios, M., Bertucci, F., et al. (2010) Discontinuation of Imatinib in Patients with Advanced Gastrointestinal Stromal Tumors after 3 Years of Treatment: An Open-Label Multicenter Randomized Phase 3 Trial. The Lancet Oncology, 11, 942-949. https://doi.org/10.1016/S1470-2045(10)70222-9

[12] Seesing, M.F.J., Tielen, R., Van Hillegersberg, R., Van Coevorden, F., Jong, K.P., Nagtegaal, I.D., et al. (2016) Resection of Liver Metastases in Patients with Gastrointestinal Stromal Tumors in the Imatinib Era: A Nationwide Retrospective Study. European Journal of Surgical Oncology, 42, 1407-1413.

https://doi.org/10.1016/j.ejso.2016.02.257 